Europäischer Fahrplan für eine $\mathrm{CO}_{2}$-arme Wirtschaft

\section{Jeder Sektor nach seinen Möglichkeiten}

Um langfristig einen gefährlichen Klimawandel zu vermeiden und die Erderwärmung auf unter zwei Grad Celsius zu begrenzen, ist die Reduktion der Treibhausgasemissionen notwendig. Die Europäische Union hat nun erste Ansätze aufgezeigt, wie dieses Ziel zu erreichen ist, ohne die Wettbewerbsfähigkeit der europäischen Wirtschaft zu gefährden. Von Jan Nill

D er Europäische Rat hat mehrfach das Ziel bestätigt, die Treibhausgasemissionen (THG) der Europäischen Union (EU) bis 2050 um 80 bis 95 Prozent gegenüber $1990 \mathrm{zu}$ reduzieren. Die internationalen Klimaschutzvereinbarungen von Kopenhagen und Cancún enthalten eine Verpflichtung zu langfristigen Strategien für eine kohlenstoffdioxidarme Entwicklung. Im März diesen Jahres hat die Europäische Kommission einen Fahrplan für die Umgestaltung der EU in eine wettbewerbsfähige kohlenstoffdioxidarme Wirtschaft bis 2050 vorgelegt (1). Dessen Ziel ist es aufzuzeigen, wie sich der EU-Politikrahmen in den kommenden zehn Jahren und danach entwickeln sollte, um

Abbildung 1: Wege zur Verringerung der THG-Emissionen in der EU um $80 \%(100 \%=1990)$

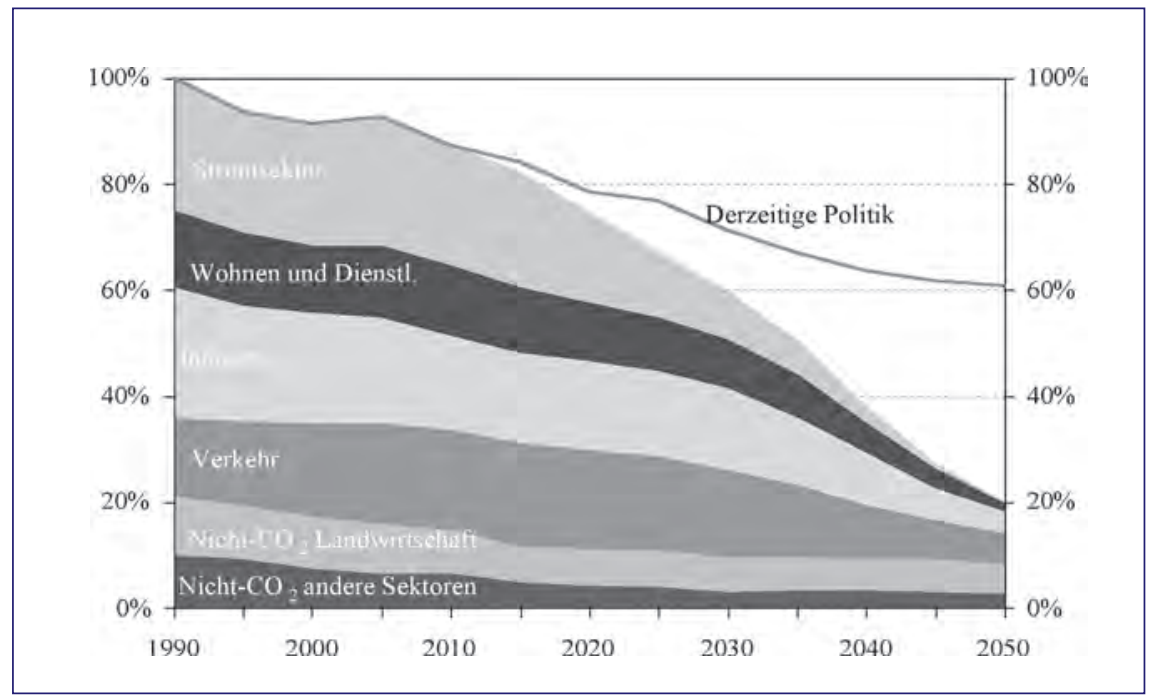

Quelle: KOM(2011) 112
- den wissenschaftlichen Erkenntnissen entsprechende, umfangreiche Senkungen der THG-Emissionen zu ermöglichen und gleichzeitig

I die Anfälligkeit für Ölschocks und andere Gefährdungen der Energieversorgungssicherheit zu verringern und

- die Chancen für nachhaltige Technologieentwicklung und Beschäftigung zu nutzen, ohne weitergehende Überlegungen der Nachhaltigkeit und Ressourceneffizienz zu vernachlässigen.

Auf der Basis einer Vielzahl modellbasierter Szenarioanalysen zeichnet der Fahrplan einen kostengünstigen Weg zur Erreichung dieser Ziele vor (2). Bis 2050 sollte die EU allein durch EU-interne Maßnahmen die Emissionen um 80 Pro-

zent gegenüber 1990 reduzieren, und nur für darüber hinausgehende Emissionsminderungen auf internationale Märkte für Kohlenstoffdioxid $\left(\mathrm{CO}_{2}\right)$ zurückgreifen. Solche Emissionsminderungen sind ausgehend von bestehenden Technologien möglich, sofern ein hinreichend starker $\mathrm{CO}_{2}$-Preisanreiz in allen Sektoren gilt (2050 zwischen etwa 100 Euro und 370 Euro je Tonne $\mathrm{CO}_{2}$-Äqu). Als Zwischenetappen müssen die internen Emissionen bis 2030 um etwa 40 Prozent und bis 2040 um etwa 60 Prozent gegenüber 1990 verringert werden. Die Analyse zeigt auch, dass der kostengünstigste Weg zum Emissionsminderungsziel bis 2020 eine EU interne Reduzierung um 25 Prozent beinhaltet. Dies kann erreicht werden, sofern die EU ihr Ziel einer 20-prozentigen Verbesserung der Energieeffizienz bis 2020 verwirklicht.

Alle Sektoren werden einen erheblichen Beitrag leisten müssen (siehe Abbildung 1). Bei gleichen wirtschaftlichen Anreizen für Emissionsverringerungen in allen Sektoren wäre ein größerer Beitrag der Sektoren, die unter das EU-Emissionshandelssystem (EHS) fallen, weiterhin kosteneffektiv. In den EHS-Sektoren würden Emissionssenkungen um knapp 45 Prozent (2030) beziehunsgweise rund 90 Prozent (2050) gegenüber 2005 erzielt werden, während die nicht unter das EHS fallenden Sektoren ihre Emissionen bis 2030 um etwa 25 Prozent und bis 2050 um knapp 70 Prozent im Vergleich zu 2005 reduzieren würden.

Die höchsten kosteneffektiven Emissionssenkungen ergäben sich im Stromsektor. Dieser würde den $\mathrm{CO}_{2}$-Ausstoß aufgrund der Verbreitung $\mathrm{CO}_{2}$-armer Technologien und der steigenden Effizienz auf der Nachfrageseite rapide verringern und dürfte bis 2030 Emissionssenkungen um 60 Prozent verwirklichen. Im Jahr 2050 werden im Stromsektor praktisch keine $\mathrm{CO}_{2}$-Emissionen mehr freigesetzt.

Verkehr und Landwirtschaft sind die wichtigsten Bereiche, in denen bei vergleichbaren wirtschaftlichen Anreizen in allen Sektoren auch langfristig keine völlige Dekarbonisierung zu erwarten ist. Das hieße zugleich, dass im Jahr 2050 je- 
weils ein Drittel der Gesamtemissionen der EU auf den Verkehr und die Landwirtschaft entfallen. Im Verkehrswesen wird der Wachstumstrend der letzten 20 Jahre umgekehrt. Der größte Teil der Emissionssenkungen würde zwischen 2030 und 2050 erzielt und dürfte im Verkehrssektor etwa minus 60 Prozent betragen. Für die Landwirtschaft verläuft die Kurve umgekehrt. Hier werden zwischen heute und 2030 beträchtliche Emissionssenkungen erreicht, danach allerdings kommt es auch aufgrund der wachsenden Weltbevölkerung und Ernährungsnachfrage nur noch zu geringen technischen THG-Emissionsverringerungen. Wie in anderen Sektoren ist es allerdings lohnenswert zu untersuchen, wie sich nicht-preisinduzierte Verhaltensänderungen auf Optionen für eine weitere THGEmissionsreduzierung auswirken.

Schließlich ist darauf hinzuweisen, dass zwischen den Sektoren Energie, Forstwirtschaft und Landwirtschaft komplexe und zum Teil noch unsichere Wechselwirkungen bestehen, sowohl auf europäischer als auch auf globaler Ebene. Die Nachfrage nach Bioenergie in der EU kann teilweise auch durch Einfuhren gedeckt werden, wodurch die Folgen die EU weniger, Drittländer dafür potenziell mehr betreffen würden. Hier sind weitere Untersuchungen notwendig.

Der Aufbau einer $\mathrm{CO}_{2}$-armen EUWirtschaft wird in den nächsten 40 Jahren zusätzlich zu den derzeitigen Investitionen von 19 Prozent des Bruttoinlandsprodukts (BIP) weitere jährliche Investitionen in Höhe von durchschnittlich 1,5 Prozent des EU-BIP (das sind 270 Milliarden Euro) erfordern. Durch niedrigere Ausgaben für Öl- und Gasimporte werden diese zusätzlichen Investitionen zu einem großen Teil oder gar vollständig wieder zurückgewonnen. Diese Einsparungen werden auf jährlich 175 bis 320 Milliarden Euro geschätzt. Solche Investitionen würden nicht nur die Abhängigkeit der EU von Energieimporten vermindern, sodass die EU weniger anfällig für mögliche Ölpreisschocks wäre, sie würden auch neue Quellen für Wachstum stimulieren, bestehende Arbeitsplätze erhalten und neue schaffen. Die
Luftverschmutzung und die damit verbundenen Kosten im Gesundheitswesen würden ebenfalls reduziert, was bis 2050 zu Einsparungen von insgesamt bis zu 88 Milliarden Euro pro Jahr führen könnte.

Wie geht es weiter? Die Kommission will auf der Grundlage des Fahrplans sektorspezifische politische Initiativen und Fahrpläne erarbeiten. Ein Weißbuch zum Verkehr das sich an den sektoralen Klimaschutz-Etappenzielen orientiert wurde Ende März vorgelegt (3). Ein Energiefahrplan bis 2050 ist für Ende diesen Jahres vorgesehen. Die Kommission wird außerdem weiterhin dafür sorgen, dass das EU-EHS ein Schlüsselinstrument bleibt, das bewirkt, dass kostengünstig in eine Verminderung der $\mathrm{CO}_{2}$-Emissionen investiert wird. Als Teil des Ende Juni vorgelegten Vorschlags für den Finanzrahmen des EU-Haushalts 2014-2020 plant sie zudem, mit Hilfe von Beiträgen aus verschiedenen Politikbereichen den Anteil für klimabezogene Ausgaben auf mindestens 20 Prozent anzuheben (4). Und schließlich sind die EU-Mitgliedstaaten gefragt, nationale und regionale Strategien zur Verwirklichung einer $\mathrm{CO}_{2}$-armen Wirtschaft bis $2050 \mathrm{zu}$ entwickeln.

\section{Anmerkungen}

(1) Europäische Kommission: Fahrplan für den Übergang zu einer wettbewerbsfähigen $\mathrm{CO}_{2}$ armen Wirtschaft bis 2050. KOM(2011) 112, Brüssel, den 8.3.2011. Internet: http://ec.europa.eu/clima/policies/roadmap/index_en.htm.

(2) Für eine ausführliche Darstellung vgl. die begleitende Folgenabschätzung, SEK(2011)288 und auch auf deutsch verfügbare Zusammenfassung SEK(2011)289. Internet: http://eurlex.europa.eu/Result.do?T1=V7\&T2=2011\&T3 $=289 \&$ RechType $=$ RECH_naturel $\&$ Submit $=$ Suche.

(3) $\operatorname{KOM}(2011) 144$, Internet: http://ec.europa.eu/transport/ strategies/2011_white_paper_en.htm.

(4) $\mathrm{KOM}(2011) 500 / I$ und II, Internet: http://ec.europa.eu/budget/reform.

\section{AUTOR + KONTAKT}

Dr. Jan Nill ist Referent für die ökonomische Analyse von EU Klima- und Energiepolitiken in der Europäischen Kommission.

Europäische Kommission, GD Klimaschutz, Referat CLIMA A.4. Tel.: +32 22990190 , E-Mail: Jan.Nill@ec.europa.eu

\section{soziale technik}

Seit 20 Jahren ist SOZIALE TECHNIK - herausgegeben vom Interuniversitären Forschungszentrum für Technik, Arbeit und Kultur (IFZ) - ein Forum für die interdisziplinäre Technikforschung und liefert Beiträge für eine sozial- und umweltverträgliche Gestaltung von Technologien. SOZIALE TECHNIK erscheint vierteljährlich, ein Jahresabonnement kostet $€ 20$,- (für Studierende $€ 15$,-).

Kostenloses Probeabonnement!

\section{Mit Beiträgen zu:}

$\square$ Technologie \& Politik
$\square$ Umwelt \& Energie
$\square$ Neve Biotechnologien
$\square$ Fraven \& Technik
$\square$ Informations- \&

Kommunikationstechnologien
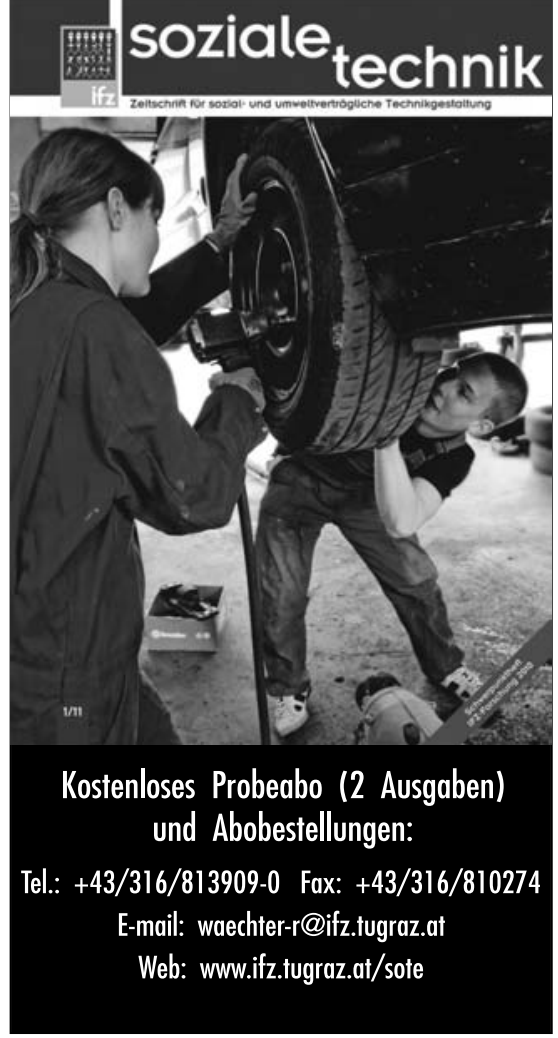


\section{Lizenzhinweis}

Die Beiträge in ÖkologischesWirtschaften werden unter der Creative-Commons-Lizenz "CC 4.0 Attribution Non-Commercial No Derivatives" veröffentlicht. Im Rahmen dieser Lizenz muss der Autor/Urheber stets genannt werden, das Werk darf nicht bearbeitet, abgewandelt oder in anderer Weise verändert und außerdem nicht kommerziell genutzt werden. Die digitale Version des Artikels bleibt für zwei Jahre Abonnent/innen vorbehalten und ist danach im Open Access verfügbar. 